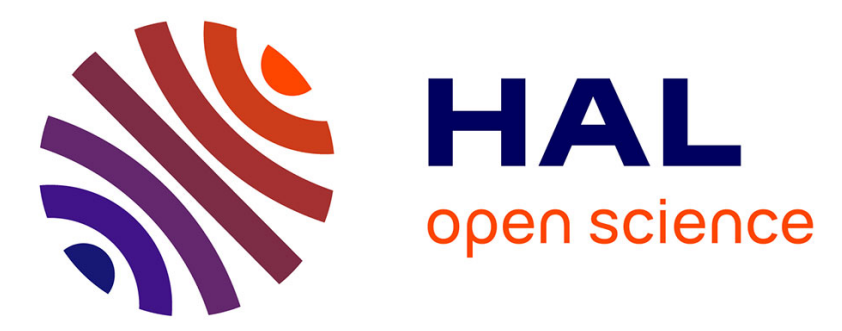

\title{
Thrombin generation test: A reliable tool to evaluate the pharmacodynamics of vitamin $K$ antagonist rodenticides in rats
}

Georges Jourdi, Sebastien Lefebvre, Bernard Le Bonniec, Emmanuel Curis, Pascale Gaussem, Virginie Lattard, Virginie Siguret

\section{To cite this version:}

Georges Jourdi, Sebastien Lefebvre, Bernard Le Bonniec, Emmanuel Curis, Pascale Gaussem, et al.. Thrombin generation test: A reliable tool to evaluate the pharmacodynamics of vitamin $\mathrm{K}$ antagonist rodenticides in rats. Pesticide Biochemistry and Physiology, 2018, 146, pp.19 - 24. 10.1016/j.pestbp.2018.02.004 . hal-01713957

\section{HAL Id: hal-01713957 \\ https://hal.science/hal-01713957}

Submitted on 4 Feb 2020

HAL is a multi-disciplinary open access archive for the deposit and dissemination of scientific research documents, whether they are published or not. The documents may come from teaching and research institutions in France or abroad, or from public or private research centers.
L'archive ouverte pluridisciplinaire HAL, est destinée au dépôt et à la diffusion de documents scientifiques de niveau recherche, publiés ou non, émanant des établissements d'enseignement et de recherche français ou étrangers, des laboratoires publics ou privés. 


\title{
Thrombin generation test: A reliable tool to evaluate the pharmacodynamics of vitamin $\mathrm{K}$ antagonist rodenticides in rats
}

\author{
Jourdi Georges ${ }^{1}$,Lefebvre Sebastien², Le Bonniec Bernard ${ }^{1}$, Curis Emmanuel ${ }^{3}$, Gaussem \\ Pascale ${ }^{4}$, Lattard Virginie ${ }^{2}$, Siguret Virginie ${ }^{5}$
}

\begin{abstract}
Vitamin $\mathrm{K}$ antagonist rodenticide pharmacodynamics $(\mathrm{PD})$ is studied in rodents with traditional laboratory tests. We wondered if thrombin generation test (TGT) could add value.

Difethialone $(10 \mathrm{mg} / \mathrm{kg})$ was administered per os to 97 OFA-Sprague Dawley rats. PD was studied over a $72 \mathrm{~h}$-period using the Calibrated Automated Thrombogram on platelet poor plasma before and after intoxication ( 3 female and 3 male rats for each 13 time points) and TGT parameters were compared with the prothrombin time (PT) and vitamin $\mathrm{K}$ dependent factor activities previously reported.

Following intoxication, preliminary tests evidenced rapid and full inhibition of thrombin generation triggered with 5 or $20 \mathrm{pM}$ human recombinant tissue factor. To study the evolution of TGT parameters following difethialone intake, we adapted the test by complementing intoxicated rat samples with pooled normal rat plasma $(3 / 1, \mathrm{v} / \mathrm{v})$. Adapted TGT confirmed the known higher procoagulant basal level in females compared to males through higher endogenous thrombin potential (ETP) and peak height $(\mathrm{PH})(\mathrm{p}<0.0001$ and $\mathrm{p}=0.0003$, respectively). An exponential model fitted well the PH and ETP decay after intoxication. In contrast to PT, the decreases were observed immediately following VKA intake and had comparable time to halving values: $10.5 \mathrm{~h}(95 \% \mathrm{CI}[8.2$; 13.6]) for ETP and $10.4 \mathrm{~h}(95 \% \mathrm{CI}[7.8 ; 14.1])$ for PH. The decrease of FVII and FX preceded that of PH, ETP and FII while FIX decreased later on, contributing to the severe hypo-coagulability.

We demonstrated that TGT performed in samples of intoxicated rats complemented with normal plasma is a reliable tool for evaluation of VKA rodenticide PD in rats.

\section{Keywords}

Thrombin generation test - Difethialone — Rodents

1 INSERM UMR S1140, Université Paris Descartes, Sorbonne Paris Cité, 4 avenue de l'Observatoire, 75006 Paris, France, ${ }^{2}$ USC 1233 RS2GP, VetAgro Sup, INRA, Univ Lyon, F-69280, 1, avenue Bourgelat, 69280 Marcy l'Etoile, Lyon, France, ${ }^{3}$ Laboratoire de biomathématiques et UMR S1144, Université Paris Descartes, Sorbonne Paris Cité et DBIM, Hôpital Saint-Louis, AP-HP, 4 avenue de I'Observatoire, 75006 Paris, France, ${ }^{4}$ Service d'hématologie biologique, Hôpital Européen Georges Pompidou, AP-HP et INSERM UMR S1140, Université Paris Descartes, Sorbonne Paris Cité, 4 avenue de l'Observatoire, 75006 Paris, France, ${ }^{5}$ Service d'hématologie biologique, Hôpital Lariboisière, AP-HP et INSERM UMR S1140, Université Paris Descartes, Sorbonne Paris Cité, 4 avenue de l'Observatoire, 75006 Paris, France

*Corresponding author: virginie.siguret@parisdescartes.fr
\end{abstract}

\section{Contents}

Introduction

1 Materials and methods

1.1 Animals and blood sampling . . . . . . . . . . 2

Blood sampling and preparation of pooled normal rat plasma $(\mathrm{PNrP}) \bullet$ Blood sampling and preparation of PPP from intoxicated rats

1.2 Thrombin generation test in rat PPP . . . . . . 3 CAT assay $\bullet$ Protocol

1.3 Statistical analyses
2 Results 4

2.1 Adaptation of TGT standard protocol to study difethialone PD in rats . . . . . . . . . . . .4

2.2 Changes of thrombin generation over time after difethialone per os intoxication .........4

3 Discussion 5

4 Financial support $\quad 7$

5 Acknowledgments 8

References 


\section{Introduction}

Vitamin K antagonists (VKA) have been used as rodenticides for more than sixty years ${ }^{1}$. They exert their anticoagulant effect by targeting the vitamin $\mathrm{K}$ 2,3-epoxide reductase complex subunit 1 , therefore blocking the recycling of vitamin $\mathrm{K}$ 2,3-epoxide in vitamin $\mathrm{K}$ hydroquinone ${ }^{2}$. Decreased availability of vitamin K hydroquinone, a co-substrate of the $\gamma$ carboxylase, results in hypo- $\gamma$-carboxylated vitamin $\mathrm{K}$ dependent clotting factors thus in dramatically decreased clotting activity of factors II, VII, IX and X (FII, FVII, FIX, FX) ${ }^{3,4}$.

VKA rodenticides are characterized by a wide interindividual variability of response between rats due to gender, environmental and genetic factors ${ }^{1,5,6}$. The origin of the gender differences in the response to VKA rodenticides has been reported in different rat strains: in Sprague-Dawley rats, a higher basal activity of FVII and FX has been found in females compared to males, in agreement with results of older studies in Wistar rats ${ }^{6,7}$. Besides, resistance to VKA rodenticides due to genetic factors has been reported in both genders from many countries in Europe but also in United States, Canada, Japan and Australia resulting in a serious concern for pest control $^{8-10}$ ]. Consequently, warfarin and diphacinone which had been introduced in the early 1950s, were replaced in the 70 s by more potent molecules such as difenacoum or bromadiolone, and 10years later by brodifacoum or flocoumafen. Nevertheless, genetic resistance to these compounds developed in parallel $1^{1,5,11,12}$. Difethialone was the latest VKA rodenticide developed for pest control and is currently considered as one of the most powerful for which no resistance has been described so far ${ }^{13}$.

Published data on the rodent PD profile of VKA rodenticides are scarce and report results obtained using coagulation tests of human clinical practice performed with rodent plasma samples ${ }^{5,7,14,15}$. Semi-global coagulation assays, namely prothrombin time (PT) and activated partial thromboplastin time are reliable tools for the exploration of coagulation system in rodents, especially to assess the anticoagulant resistance ${ }^{16}$. However, they mainly explore the initiation phase of coagulation, which requires a minimal amount of thrombin generated (i.e. $10-30 \mathrm{nM}$ ) necessary for clot formation ${ }^{17}$. More recently, additional global tests, among which the thromboelastometry and the thrombin generation test (TGT), have been proposed to evaluate thrombin generation beyond clot formation ${ }^{18-20}$, thus to follow rodents anticoagulation by VKA and propose a better rodenticides management. Rotational thromboelastometry (Rotem) did not provide a very good insight of coagulability in this setting since only two parameters obtained with Extem were modified in intoxicated rat Platelet Poor Plasma (PPP) with lag time higher than $8 \mathrm{~h}$ and very wide confidence ranges of their doubling time along with the relatively large sample volume needed. Consequently, PT remains the best method to monitor VKA rodenticides induced hypocoagulation $^{20}$. In TGT, the addition into plasma of a fluorescent substrate cleaved by thrombin allows measuring a fluorescence signal over time, which is not disturbed by the turbidity of a forming clot, in contrast to optical density for a chromogenic substrate ${ }^{21}$. In humans, TGT accurately evaluates coagulation disorders associated with bleeding or thrombosis. For example, TGT provides another insight on anticoagulation improving the monitoring of factor replacement therapy in hemophilic patients beyond clotting times ${ }^{22-25}$. However, this method has never been developed in rats intoxicated with VKA rodenticide in order to evaluate their impact on the coagulation system and therefore the rodenticide efficacy.

In a recent study, our group determined the pharmacokinetic/pharmacodynamic profile of difethialone in male and female Sprague-Dawley rats over a 72-hour period following difethialone intoxication using routine coagulation tests ${ }^{20}$. The aim of the present study was (i) to adapt the standard Calibrated Automated Thrombography (CAT) protocol to ensure the reliability of TGT to evaluate anticoagulant rodenticide PD in rat plasma samples and (ii) to determine the kinetic profiles of TGT parameters from individual intoxicated male or female rats and built models of TGT parameters kinetics after VKA intoxication. Finally, we compared TGT parameter decays to those of PT and vitamin K dependent clotting factor activities.

\section{Materials and methods}

\subsection{Animals and blood sampling}

All research procedures were performed according to an experimental protocol following international guidelines (86/609/EEC) and approved by the ethic committee of VetAgro Sup. A total of 97 eight-week old male and female OFASprague-Dawley rats (Charles River, l'Arbresles, France) were used in the present study. All rats were acclimatized for at least five days and housed in the animal facility, four rats per cage as already reported in details ${ }^{20}$. Just before blood sampling, rats were anesthetized with isoflurane and euthanized with $\mathrm{CO} 2$ immediately after sampling.

\subsubsection{Blood sampling and preparation of pooled normal rat plasma (PNrP)}

Since female have higher basal procoagulant level compared to male rats ${ }^{20}$, we chose to use only male rats to prepare a pool of normal rat plasma. Therefore a group of 10 male rats were fed with standard diet Scientific Animal Food and Engineering, referenced A04 (Augey, France). Blood was collected by cardiac puncture in $3.5 \mathrm{~mL}$ tubes containing sodium citrate (3.2\% 0.105M; 1:9v/v; Greiner, Alcyon, Nancy, France) and gently inverted five times to ensure adequate anticoagulation. Tubes were then double centrifuged at $2000 \mathrm{~g} 15 \mathrm{~min}$ at $20^{\circ} \mathrm{C}$ and PPP samples were pooled and served as Pooled Normal rat Plasma ( $\mathrm{PNrP})$. $\mathrm{PNrP}$ was aliquoted, frozen and stored at $-80^{\circ} \mathrm{C}$ until use. PT and aPTT results measured on PNrP were in the reference intervals ${ }^{20}$.

\subsubsection{Blood sampling and preparation of PPP from intox- icated rats}

In order to adapt the CAT protocol for intoxicated rat PPP samples, 9 male rats were fed with vitamin K3-deficient food 

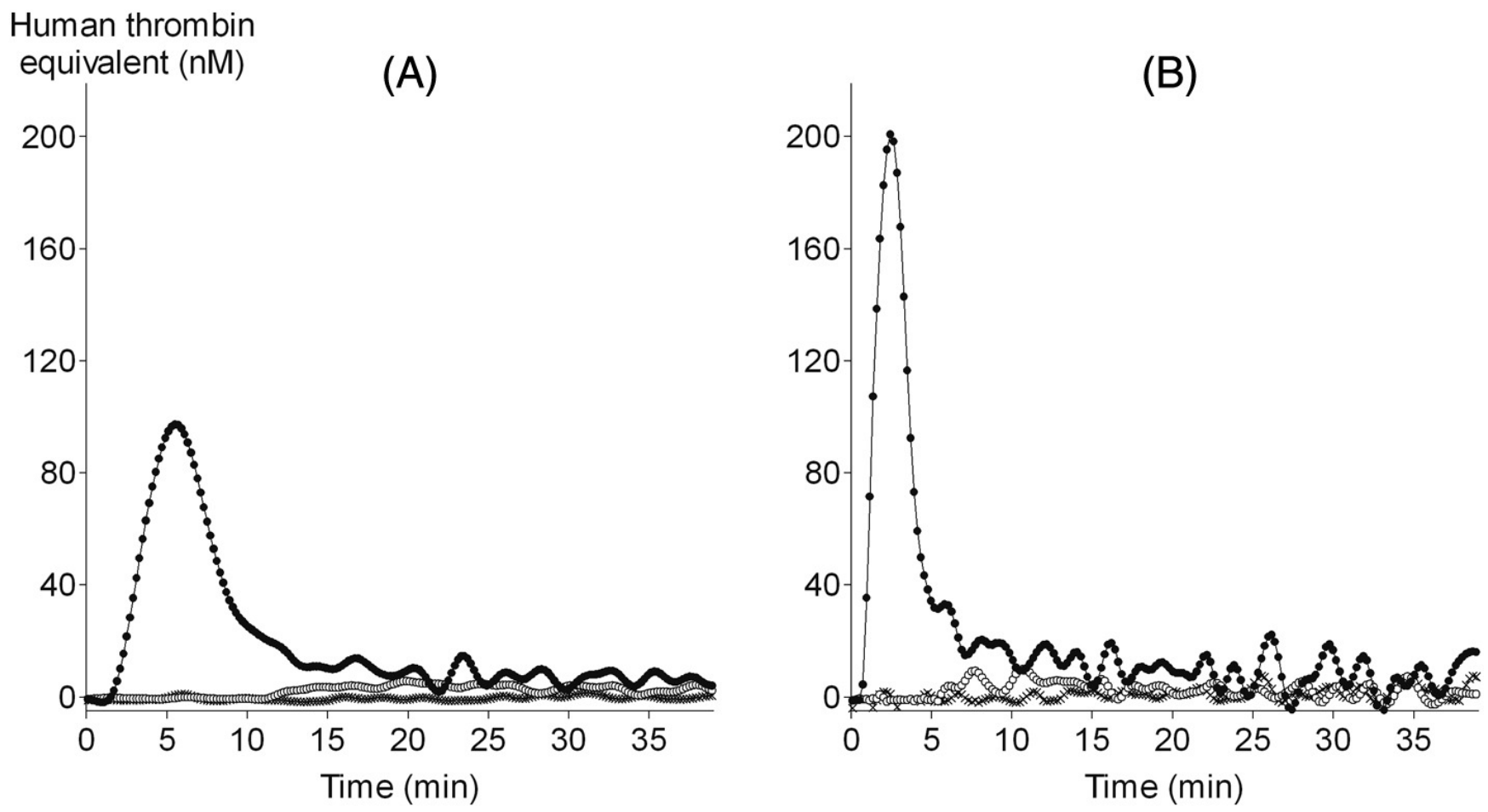

Figure 1. TGT with rat PPP. TGT were performed in pooled PPP samples from 3 male rats using the CAT standard protocol triggered with either PPP-reagent (1A) or High PPP-reagent (1B). In both panels, closed circles represent TGT performed at T0 (prior to intoxication), open circles TGT at T24 after difethialone intoxication, and crosses at T72. PH at T0 doubled whereas TTP halved when TGT was triggered with 20 instead of 5pM tissue factor. Thrombin levels are expressed as equivalent human thrombin. With both triggers, thrombin generation was completely inhibited at T24 and T72 post difethialone intoxication.

(Scientific Animal Food and Engineering), at least $48 \mathrm{~h}$ before the beginning and throughout the experience as described ${ }^{20}$. Animals received by force-feeding a per-os administration of $10 \mathrm{mg} / \mathrm{kg}$ difethialone (kindly provided by Liphatech, Pontdu-Casse, France), corresponding to 20-fold the lethal dose LD50. Pooled PPP samples from 3 male rats obtained at T0, T24 and T72 after difethialone intoxication were prepared and stored as described above.

In a second phase and in order to study coagulation system after intoxication with difethialone, 39 male and 39 female rats were force-fed as above. Groups of 3 males and 3 females were anesthetized prior to blood sampling as described above at different times (T) (in hours) after difethialone administration: T0, T1, T2, T4, T8, T10, T13, T16, T20, T24, T32, T48 and T72. All PPP samples were thawed in a $37^{\circ} \mathrm{C}$ water bath just before use.

\subsection{Thrombin generation test in rat PPP 1.2.1 CAT assay}

TGTs were performed on a CAT system according to the method described by Hemker et al. ${ }^{21}$. TGTs were measured using a microplate fluorometer (Fluoroskan Ascent ${ }^{\mathrm{TM}}$, ThermoLabsystems, Helsinki, Finland). Rodent PPP tends to have short lag time and rapid thrombin generation resulting that if all of the 96 wells of a microplate are used simultaneously, lack of the early points for the second half of the microplate precludes accurate ETP evaluation ${ }^{26}$. Using only the first half of the 96-well plate allows starting on time all fluorescence measurements following initiation. Consequently, half of a round bottom 96-well plate (Greiner, Frickenhausen, Germany) was used each time. PPP samples were run in duplicate using either $20 \mu \mathrm{L}$ PPP-reagent $(5 \mathrm{pM}$ tissue factor and $4 \mu \mathrm{M}$ phospholipid vesicles; Stago, Asnières sur Seine, France) or $20 \mu \mathrm{L}$ High PPP-reagent $(20 \mathrm{pM}$ tissue factor and $4 \mu \mathrm{M}$ phospholipid vesicles; Stago), and $20 \mu \mathrm{L}$ Thrombin Calibrator (Stago) in parallel; average of the duplicates was used for analysis and mean $\mathrm{CV}$ values varied between $1.5 \%$ for temporal TGT parameters and 3\% for the others. TGT plate containing the mixture of $80 \mu \mathrm{L} \mathrm{PPP}$ and $20 \mu \mathrm{L}$ triggering reagent or Thrombin Calibrator was incubated $5 \mathrm{~min}$ in the machine to warm to $37^{\circ} \mathrm{C}$. TGT was initiated by autodispensing $20 \mu \mathrm{L}$ of FluCa solution (Stago) containing calcium ions and the fluorogenic substrate, Z-Gly-Gly-Arg-7-amino-4-methylcoumarin, to each well. Generated thrombin cleaves the fluorogenic substrate and fluorescence increase was monitored at $37^{\circ} \mathrm{C}$ for $120 \mathrm{~min}$. Thrombinoscope software (Thrombinoscope $\mathbb{R}$ $\mathrm{BV}$, version 5.0.0.742, Maastricht, Netherlands) was used to calculate the amount of thrombin generated over time expressed as human thrombin equivalent taking into account for each sample the calibrator activity. We recorded and interpreted the following parameters: lag time (LT; in min: time from test triggering to signal detection), time to peak (TTP; 
in min: time necessary for thrombin concentration to reach its maximal value), peak height ( $\mathrm{PH}$; in $\mathrm{nM}$ : maximal human thrombin equivalent generated amount) and endogenous thrombin potential (ETP; in nM.min: area under the thrombin concentration curve).

\subsubsection{Protocol}

TGTs were first performed without complementation in pooled PPP samples from 3 male rats before (T0), and after administration of difethialone (at T24 and T72), using either PPP- or High PPP-reagent. TGT were subsequently performed in PPP samples complemented with PNrP at different ratios (PPP/PNrP; v/v) to determine the optimal complementation providing reliable results in TGT.

In a second phase of experiments, TGT triggered with the PPP-reagent were performed in female and male PPP samples obtained at various time-points post intoxication. There, samples were complemented with PNrP at the previously determined ratio of $3 / 1 \mathrm{PPP} / \mathrm{PNrP}(\mathrm{v} / \mathrm{v})$ to evaluate the kinetics of TGT parameters following VKA intoxication.

\subsection{Statistical analyses}

TGT parameters from the Thrombinoscope software were further analyzed using the R software (version 3.3.1; R Foundation for Statistical Computing, Vienna, Austria). Intraassay coefficient of variation (CV) were computed from the parameters of TGT performed in $\mathrm{PNrP}$ and triggered with PPP-reagent; their 95\% confidence intervals (95\% CI) were obtained as the 2.5 and 97.5 percentiles of their bootstrap distribution (9999 random draws).

TGT parameters, assigned as y, were modeled as a function of time $(\mathrm{t})$ after rodenticide administration, using an exponential decay model, $\mathrm{y}=\mathrm{A} \mathrm{e}-\mathrm{k} \cdot \mathrm{t}+\mathrm{B}+\varepsilon$, where $\mathrm{t}=0$ at the administration time, $\mathrm{A}$ is the basal value, $\mathrm{B}$ is the residual value of the considered parameter, $\mathrm{k}$ is the rate constant related to the half-life ( $\mathrm{t} \frac{1}{2}$ ) by the relation $\mathrm{t} \frac{1}{2}=\frac{\log (2)}{k}$, and $\varepsilon$ is an error term, assumed to be Gaussian ("normal"), centered and of constant variance. The model was fitted using non-linear leastsquares (nls function in R). Gender effect was tested using sex and A, sex and k, or sex and B interaction terms, and the resulting models were compared using nested models, with an asymptotic likelihood ratio test. Results were expressed as mean with their $95 \%$ CI obtained by profiling. Tests were considered significant if $\mathrm{p}_{i} 0.05$. Initial values for the non-linear regression were obtained, for $\mathrm{B}$, by averaging the last values, then, for $\mathrm{A}$ and $\mathrm{k}$, by a linear regression of $\log (\mathrm{y}-\mathrm{B})$ on time. Adequation of the model to the data was checked based on the fit residuals: no significant heteroskedasticity, approximate normal distribution, no clear residual curvature.

\section{Results}

\subsection{Adaptation of TGT standard protocol to study difethialone PD in rats}

TGT were first triggered with PPP-reagent ( $5 \mathrm{pM}$ tissue factor) in pooled PPP samples from 3 male rats obtained at T0,
T24 and T72. Before difethialone administration (T0), TGT resulted on the average in a thrombin generation culminating at $100 \mathrm{nM}$ PH for a $5 \mathrm{~min}$ TTP. Complete inhibition of thrombin generation was observed at both T24 and T72 post difethialone administration over the $2 \mathrm{~h}$ of fluorescence measurement (Figure 1A).

When TGT were triggered with High PPP-reagent (20pM tissue factor) the PH at T0 doubled whereas TTP halved compared to the values obtained using the PPP-reagent. Nevertheless High PPP-reagent also failed to trigger thrombin generation in the T24 and T72 samples following difethialone administration (Figure 1B).

As complete inhibition of thrombin generation was observed at T24 with pooled PPP from intoxicated rats triggered with either 5 or $20 \mathrm{pM}$ tissue factor reagent, pooled PPP at T24 was complemented with $\mathrm{PNrP}$ at different $\mathrm{PPP} / \mathrm{PNrP}$ ratios (v/v) $(9 / 1,4 / 1,7 / 3,1 / 1$ and 1/3) (Table 1). TGT performed with $\mathrm{PNrP}$ and triggered with $5 \mathrm{pM}$ tissue factor resulted in a CV of $4.6 \%$ (95\% CI $[3.7 ; 6.2])$ for TTP, 4.4\% (95\% CI [3.1; 6.3]) for PH, and 7.8\% (95\% CI [6.2; 11.2]) for ETP. Since fluorescence was not measured continuously in the Thrombinoscope, it resulted that all LT values obtained with PNrP were short and equal to $1.33 \mathrm{~min}$. As the PNrP fraction increased, PH and ETP of TGT triggered with the PPP reagent progressively increased up to the values obtained with $\mathrm{PNrP}$ alone. As little as one volume of PNrP for 9 volumes of plasma from intoxicated rats already considerably shortened LT and TTP (Figure 2). Similar results were obtained through PNrP complementation of PPP collected at T72 (data not shown). The 3/1 PPP/PNrP ratio was found optimal to obtain reliable TGT parameters, especially PH and ETP. Such complementation therefore allowed studying the $72 \mathrm{~h}$ kinetic of TGT parameters following per os administration of the rodenticide.

\subsection{Changes of thrombin generation over time after difethialone per os intoxication}

TGTs were then performed in individual PPP samples from male and female rats obtained at the 13 pre-specified time points over a 72h-period following difethialone intoxication. Each sample was complemented (PPP/PNrP 3/1). During the 72h-period and in both genders, PH and ETP decreased up to a similar plateau value (Figure $3 \mathrm{~A}$ and B) whereas, LT and TTP were not significantly modified (Figure 3C and D).

Different PD models were built taking into account the complementation with PNrP. Regarding ETP and PH, the exponential decay models fitted well the experimental data in males as well as in females and over than $80 \%$ of the total variance are explained by the models (Table 2). Before intoxication (T0), the basal PH values estimated by the model (parameter A) were significantly higher in females $(94 \mathrm{nM}$ 95\% CI [82; 107]) than in males (70nM [57; 83], $\mathrm{p}=0.0003$; Figure $3 \mathrm{~A})$. The same observation held for estimated basal ETP (352 [315; 390] vs 253nMmin [213; 295], pi 0.0001 ; Figure $3 \mathrm{~B}$ ). After intoxication, rat gender did not signifi- 


\begin{tabular}{|c|c|c|c|}
\hline & LT (min) & ETP (nMmin) \\
\hline \multicolumn{2}{|l|}{$\mathrm{PNrP}$} & 2.8 & 301 \\
\hline \multicolumn{3}{|l|}{$(\mathrm{v} / \mathrm{v})$} & 234 \\
\hline \multicolumn{4}{|l|}{$(\mathrm{v} / \mathrm{v})$} \\
\hline \multicolumn{4}{|l|}{$(\mathrm{v} / \mathrm{v})$} \\
\hline \multicolumn{4}{|l|}{$\begin{array}{l}\text { PPP/PNrP } \\
(\mathrm{v} / \mathrm{v})\end{array}$} \\
\hline \multicolumn{4}{|l|}{$\begin{array}{l}\text { PPP/PNrP } \\
(\mathrm{v} / \mathrm{v})\end{array}$} \\
\hline \multicolumn{2}{|c|}{$\begin{array}{l}\text { PPP non comple- } \\
\text { mented }\end{array}$} & $\begin{array}{l}\text { Below } \\
\text { the LOQ }\end{array}$ & $\begin{array}{l}\text { Below } \\
\text { the LOQ }\end{array}$ \\
\hline
\end{tabular}

Table 1. Pooled PPP samples of 3 male rats obtained 24h after difethialone intoxication were tested by TGT with or without complementation with pooled normal rat plasma (PNrP) at different PPP/PNrP ratios (v/v) (9/1, 4/1, 7/3, 1/1 and $1 / 3)$. TGT were triggered with PPP reagent $(5 \mathrm{pM}$ tissue factor). LT corresponds to the time necessary to reach $10 \mathrm{nM}$ human thrombin equivalent and ETP the area under thrombin concentration curve following coagulation activation. As $\mathrm{PNrP}$ fraction increased, LT values progressively decreased while ETP increased up to reach that of PNrP alone.

cantly influence the k parameter in both PH and ETP models ( $p=0.1808$ and $p=0.1922$, respectively). No significant differences were observed between males and females on the residual values of $\mathrm{PH}$ and ETP (parameter $\mathrm{B} ; \mathrm{p}=0.88$ and $\mathrm{p}=0.94$, respectively).

Consequently, times to halving PH and ETP were comparable in males and females $(\mathrm{p}=0.1808$ and $\mathrm{p}=0.1922$, respectively), leading to values of $10.4 \mathrm{~h}(95 \% \mathrm{CI}[7.8 ; 14.1])$ for $\mathrm{PH}$ and $10.5 \mathrm{~h}(95 \% \mathrm{CI}[8.2 ; 13.6])$ for ETP (Table 3$)$.

Half-lives of the vitamin $\mathrm{K}$ dependent factors following difethialone intoxication had been previously estimated in aliquots collected during this same study ${ }^{7}$ and reported: $2.9 \mathrm{~h}$ for FVII, 5.5h for FX, 11h for FII and 12.6h for FIX. Based on the time to halving values, ETP and PH were ranked: in both genders, FVII decreased first, followed by FX, then ETP, $\mathrm{PH}$, and FII then finally by FIX.

\section{Discussion}

In the present study, we adapted and validated the CAT as a reliable TGT method to evaluate the PD of VKA rodenticides in intoxicated rats. TGT had been proposed previously as a complementary approach to study coagulation system in rodents ${ }^{14,19,27-30}$, but, to our knowledge, this method had never been used on rodents plasma after VKA intoxication.

TGT has the advantages to globally study the coagulation system including initiation, amplification and propagation phases, resulting in large amounts of generated thrombin overtime controlled by physiological inhibitors albeit TGT performed without the addition of thrombomodulin did not

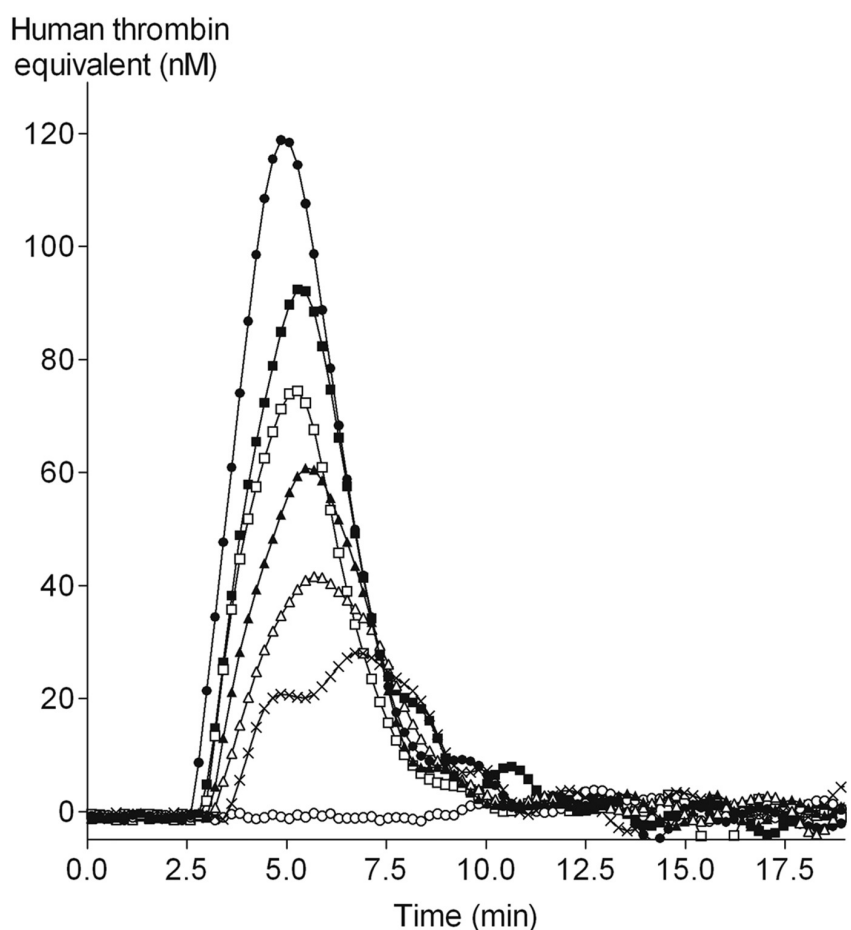

Figure 2. TGT with complemented rat PPP. Pooled PPP samples from 3 male rats at $\mathrm{T} 24$ after difethialone intoxication were complemented with $\mathrm{PNrP}$ at different ratios: PNrP only (closed circles), PPP/PNrP 1/3 (v/v) (closed squares), 1/1 (open squares), 7/3 (closed triangles), 4/1 (open triangles), 9/1 (crosses) and no complementation (open circles). Thrombin levels are expressed as equivalent human thrombin. Highest complementation (ratio 1/3) restored PH and ETP just below the values observed with PNrP alone. Lowest complementation (ratio 9/1) already considerably decreased LT and TTP likely by restoring the initiation phase of coagulation.

allow exploring the protein $\mathrm{C}$ system. Hyper- as well as hypocoagulability states including responsiveness to anticoagulants might be explored reliably through TGT parameters ${ }^{31}$. Data derived from thrombinography can be used to determine temporal parameters (LT and TTP) as well as thrombin generated (PH and ETP), useful parameters for assessing coagulation state $^{32}$.

In a paper recently published by our group regarding the PD of difethialone (7), traditional semi-global coagulation tests such as PT were used besides coagulation factor activity measurement. However, PT has some limitations. In PT test, coagulation is triggered with calcified thromboplastin reagent containing large amounts of tissue factor resulting in clot formation as soon as $10-30 \mathrm{nM}$ of thrombin are generated, thus limiting the PT exploration to the initiation phase of coagulation $^{31}$. Moreover, the large excess of tissue factor contained in thromboplastin reagent hardly encountered reallife patho-physiological situations. Appropriate laboratory tests sensitive to early, transient as well as long lasting hypo- 

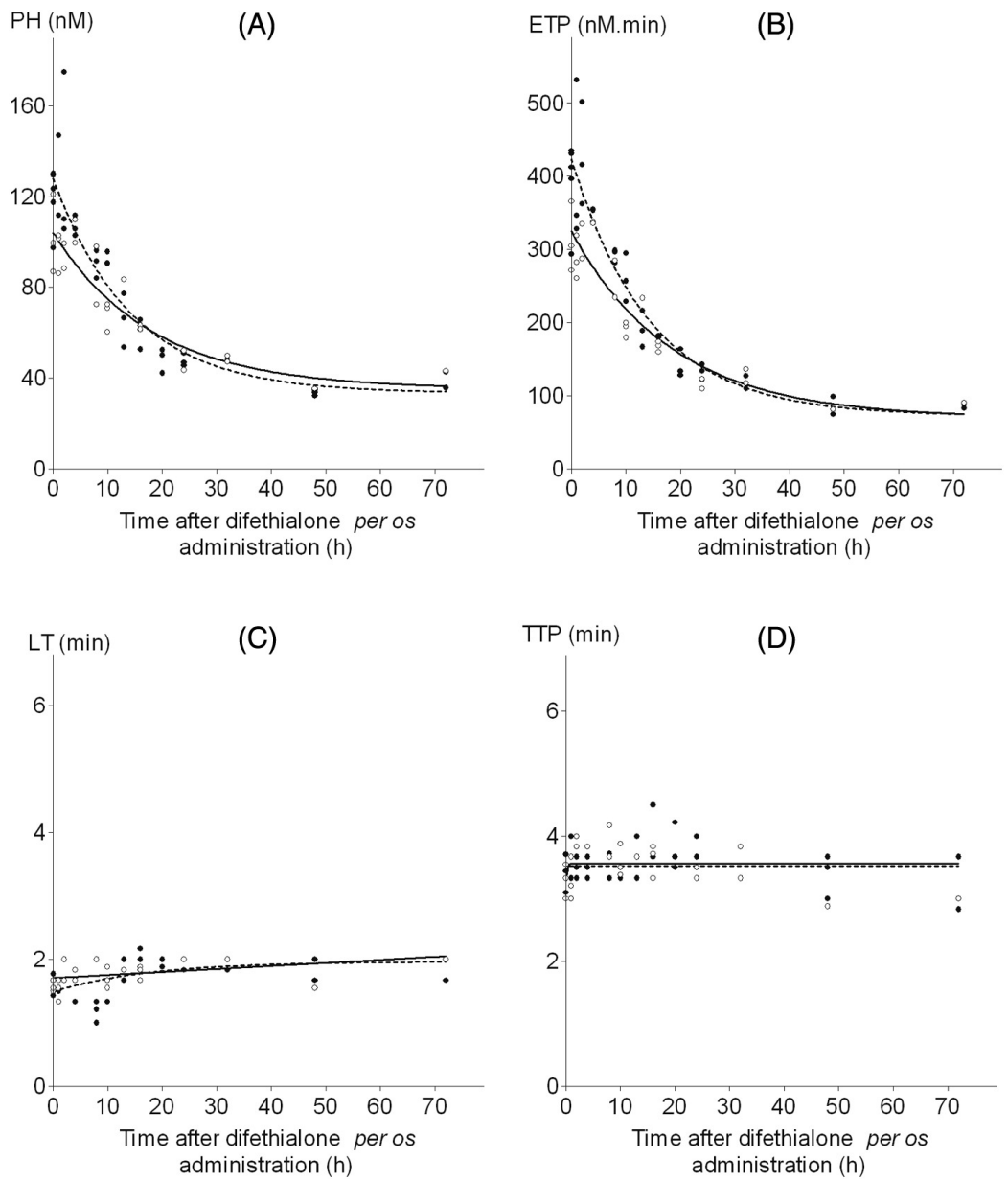

Figure 3. Changes of thrombin generation parameters over time after difethialone intoxication: PH (3A), ETP (3B), LT (3C) and TTP (3D). Graph represents the resulting parameters of TGT performed with PPP reagent as a function of the time (in hours) following intoxication (T0, T1, T2, T4, T8, T10, T13, T16, T20, T24, T32, T48 and T72). The PPP/PNrP ratio was 3/1 (v/v). Male and female individuals are represented by open and closed circles, respectively. PH (panel 3A) and ETP (panel 3B) were significantly higher at $\mathrm{T} 0$ in females than in males $\left(\mathrm{p}=0.0015\right.$ and $\mathrm{p}_{i} 0.0001$, respectively). Both decreased progressively after difethialone intoxication Time to halving ETP and PH values did not differ significantly between males and females $(\mathrm{p}=0.1922$ and $\mathrm{p}=0.1808$, respectively). Same residual values of PH and ETP were observed, owing to PNrP used for PPP complementation in both groups. LT (panel 3C) and TTP (panel 3D) did not change over time reflecting the sufficient amount of clotting factors added by PPP complementation with PNrP to normally initiate coagulation.

or hyper-coagulation would be ideal tools in VKA rodenticide development strategy. Yet PT responds only partially to this challenge since in rats it pictures hypo-coagulation with a delay ( $2 \mathrm{~h}$ in male and 8 in female rats) rapidly decreasing to undetectable level thereafter.

For all these reasons, we chose to perform TGT with rat PPP after VKA rodenticide intoxication, namely, difethialone in the present study. This rodenticide was chosen because it is known as one of the most potent inhibitor of vitamin $\mathrm{K}$ 2,3-epoxide reductase complex subunit 1 and that to date no resistance to difethialone has been reported in rodents. The dose of $10 \mathrm{mg} / \mathrm{kg}$ used in the protocol corresponds to nearly 20 fold the lethal dose $50^{33}$ ensuring that all vitamin $\mathrm{K}$ dependent factors are not functional. It is worth to note that unlike in humans and mice, in rats, proteins induced by vitamin $\mathrm{K}$ antagonists are predominantly retained in the liver and thus are not released into the bloodstream ${ }^{34}$. Consequently they do not affect TGT in VKA intoxicated rats.

Since thrombin generation was completely inhibited at T24 and T72 after difethialone intoxication, even when triggered with high tissue factor concentration $(20 \mathrm{pM})$, we successfully adapted TGT protocol by complementation of the samples with PNrP. Functional $\gamma$-carboxylated factors brought by $\mathrm{PNrP}$ restored in part thrombin generation and thus allowed studying the kinetics of two of the TGT parameters, namely $\mathrm{PH}$ and ETP.

At baseline, TGT performed in complemented PPP samples confirmed a significant higher hypercoagulability state in female compared to male rats, as reflected by significantly higher ETP and PH. This gender difference is in agreement 


\begin{tabular}{|c|c|c|c|c|c|c|}
\hline Rats & $\begin{array}{l}\text { female } \\
(\mathrm{n}=39)\end{array}$ & & $\begin{array}{l}\text { male } \\
(\mathrm{n}=39)\end{array}$ & & $\begin{array}{l}\text { Residual } \\
\text { error }\end{array}$ & $\begin{array}{l}\text { Explained } \\
\text { variance }\end{array}$ \\
\hline Parameters & A & B & A & B & & \\
\hline $\mathrm{PH}$ & $\begin{array}{l}94.5 \\
{[81-108]}\end{array}$ & $\begin{array}{lc}0.069 & 33.4 \\
{[0.050-0.095][21.1-43.8]}\end{array}$ & $\begin{array}{l}68.9 \\
{[49.7-98.3]}\end{array}$ & $\begin{array}{lc}0.055 & 35.2 \\
{[0.024-0.106][2.9-52.34]}\end{array}$ & 13.1 & $83.1 \%$ \\
\hline ETP & $\begin{array}{l}352 \\
{[311-393]}\end{array}$ & $\begin{array}{lc}0.069 & 72.4 \\
{[0.052-0.091][33.5-105.8]}\end{array}$ & $\begin{array}{l}255 \\
{[194-344]}\end{array}$ & $\begin{array}{lc}0.054 & 69.5 \\
{[0.027-0.094][26.7-125.5]}\end{array}$ & 40.9 & $87.5 \%$ \\
\hline
\end{tabular}

Table 2. Pooled PPP samples of 3 male rats obtained $24 \mathrm{~h}$ after difethialone intoxication were tested by TGT with or without complementation with pooled normal rat plasma (PNrP) at different PPP/PNrP ratios (v/v) (9/1, 4/1, 7/3, 1/1 and 1/3). TGT were triggered with PPP reagent (5pM tissue factor). LT corresponds to the time necessary to reach 10nM human thrombin equivalent and ETP the area under thrombin concentration curve following coagulation activation. As PNrP fraction increased, LT values progressively decreased while ETP increased up to reach that of $\mathrm{PNrP}$ alone.

\begin{tabular}{cllll}
\hline Parameters & $\begin{array}{l}\text { Times to halving } \\
\text { (hours) }[95 \% \mathrm{CI}]\end{array}$ & & & \\
& Both genders $(\mathrm{n}=78)$ & Females $(\mathrm{n}=39)$ & Males $(\mathrm{n}=39)$ & p value \\
\hline ETP & $10.5 \mathrm{~h}[8.2 ; 13.6]$ & $10.1[7.8 ; 13.1]$ & $12.7[8.7 ; 18.9]$ & 0.1922 \\
PH & $10.4 \mathrm{~h}[7.8 ; 14.1]$ & $9.9[7.4 ; 13.5]$ & $13.1[8.4 ; 20.6]$ & 0.1808 \\
\hline
\end{tabular}

Table 3. Time to halving ETP and PH after difethialone intoxication of male and female rats. Times to halving ETP and PH were calculated using $\mathrm{k}$ value of each exponential decay model. Mean with the $95 \% \mathrm{CI}$ were reported for female and male rats and showed no significant difference between genders $(\mathrm{p}=0.1922$ for $\mathrm{ETP}$ and $\mathrm{p}=0.1808$ for $\mathrm{PH})$. Common value was consequently estimated at $10.5 \mathrm{~h}$ for ETP and $10.4 \mathrm{~h}$ for PH after VKA intoxication. $\mathrm{p}$ value : Comparison between females and males.

with previous results obtained by our group using PT, activated partial thromboplastin time and clotting factor activities (FVII, FX) measurements. Early after intoxication, thrombin generation rapidly decreased over time resulting in an exponential decay of PH and ETP, in both genders. Experimental data fitted well with the decay models as shown by $>80 \%$ of the total variance explained by the models. On the opposite, we have already reported that following intoxication PT significantly increases only after remaining unchanged for $2 \mathrm{~h}$ in males and $8 \mathrm{~h}$ in females [7]. Our study therefore outlines the low sensitivity of TP compared to TGT for an early detection of hypo-coagulability in rats.

Interestingly, ranking ETP and PH parameters along with vitamin $\mathrm{K}$ dependent factor decays based on the time to halving values suggested that ETP and PH mostly reflected FII level. Since FVII and FX added by PNrP complementation already normalized LT and TTP, they could not further decrease ETP and PH. TGT is a valuable tool allowing PD evaluation of VKA rodenticides in one test instead of a panel of four single clotting factor activity assays. Noteworthy, PH and ETP reached a plateau around $48 \mathrm{~h}$ after intoxication, corresponding to the effect of the functional factor amounts brought by the added PNrP at a fixed ratio. Since VKA indirectly exert their anticoagulant effect by targeting vitamin $\mathrm{K}$ 2,3-epoxide reductase complex subunit 1 , they do not inhibit functional factors added by the complementation, thus not hampering the kinetics study.

Our study has some limitations. Firstly, intoxicated rat samples were complemented with PNrP obtained from only male rats. Female rats are known to be hypercoagulable, therefore this complementation in $\mathrm{PNrP}$ from male rats might reduce the difference in basal coagulation state between the two genders. Yet ETP and PH values at T0 remained significantly higher in female compared to male rats, thus these results suggest that our TGT protocol is well adapted to study the coagulability state in rats. Secondly, temporal parameters of the TGT, i.e. LT and TTP, remained stable over time probably due to $\mathrm{PNrP}$ complementation providing sufficient amounts of functional factors to restore clotting initiation, thus making their interpretation inadequate, unlike ETP and PH. Thirdly, in the present paper, we studied difethialone as rodenticide; however, we can reasonably consider that the adapted TGT can be applied to the PD study of other VKA rodenticides since they all inhibit vitamin $\mathrm{K} 2,3$-epoxide reductase complex subunit 1 and that difethialone is one of the most potent inhibitor recently developed for rat control.

In conclusion, we propose TGT as a reliable additional tool to evaluate rodenticide PD and efficacy in rodents control strategy. TGT allows global evaluation of hypercoagulability state at baseline as well as early evaluation of VKA rodenticide anticoagulant effect in rats contrary to traditional tests.

\section{Financial support}

This work was supported by grants ISI n ${ }^{\circ} 1301001 \mathrm{~W}$ "NEORAMUS" from Bpi France and by Conny-Maeva charitable Foundation (2014). 


\section{Acknowledgments}

We thank V. Joubert and C. Lagarrigue for the excellent technical assistance.

\section{References}

[1] Hans-Joachim Pelz et al. "The Genetic Basis of Resistance to Anticoagulants in Rodents". In: Genetics 170.4 (Aug. 2005), pages 1839-1847. ISSN: 0016-6731. DOI: $10.1534 /$ genetics.104.040360 (cited on page 2).

[2] Johannes Oldenburg et al. "Vitamin K Epoxide Reductase Complex Subunit 1 (VKORC1): The Key Protein of the Vitamin K Cycle". In: Antioxid. Redox Signal. 8.3-4 (2006 Mar-Apr), pages 347-353. ISSN: 15230864. DOI: 10.1089 / ars . 2006.8 .347 . pmid: 16677080 (cited on page 2).

[3] Tao Li et al. "Identification of the Gene for Vitamin K Epoxide Reductase". In: Nature 427.6974 (Feb. 5, 2004), pages 541-544. ISSN: 1476-4687. DOI: 10. 1038 / nature02254. pmid: 14765195 (cited on page 2).

[4] Simone Rost et al. "Mutations in VKORC1 Cause Warfarin Resistance and Multiple Coagulation Factor Deficiency Type 2". In: Nature 427.6974 (Feb. 5, 2004), pages 537-541. ISSN: 1476-4687. DOI: $10.1038 /$ nature02214. pmid: 14765194 (cited on page 2).

[5] J. Erica Gill et al. "Blood-Clotting Response Test for Bromadiolone Resistance in Norway Rats". In: The Journal of Wildlife Management 58.3 (July 1, 1994), pages 454-461. ISSN: 0022-541X. DOI: 10 . 2307 / 3809316. JSTOR: 3809316 (cited on page 2).

[6] Gerard M Kerins and Alan D MacNicoll. "Comparison of the Half-Lives and Regeneration Rates of Blood Clotting Factors II, VII, and X in AnticoagulantResistant and Susceptible Norway Rats (Rattus Norvegicus Berk.)" In: Comparative Biochemistry and Physiology Part C: Pharmacology, Toxicology and Endocrinology 122.3 (Mar. 1999), pages 307-316. ISSN: 0742-8413. DOI: 10 . 1016/S0742-8413 (98) 10128-7 (cited on page 2).

[7] Sébastien Lefebvre et al. "Origin of the Gender Differences of the Natural Resistance to Antivitamin K Anticoagulants in Rats". In: Toxicology (Feb. 6, 2016). ISSN: 1879-3185. DOI: $10.1016 / j$. tox . 2016. 02.002 . pmid: 26860702 (cited on pages 2, 5).

[8] W.B. Jackson and A.D. Ashton. "Case Histories of Anticoagulant Resistance". In: Pesticide Resistance: Strategies and Tactics for Management (1986), pages 355-369 (cited on page 2).
[9] A. Myllymäki. "Anticoagulant Resistance in Europe: Appraisal of the Data from the 1992 EPPO Questionnaire". In: Pesticide Science 43.1 (1995), pages 69-72. DOI: $10.1002 / \mathrm{ps} .2780430111$ (cited on page 2).

[10] Hans-Joachim Pelz et al. "Distribution and Frequency of VKORC1 Sequence Variants Conferring Resistance to Anticoagulants in Mus Musculus". In: Pest. Manag. Sci. 68.2 (2012), pages 254-259. ISSN: 1526-4998. DOI: 10.1002 /ps.2254 (cited on page 2).

[11] F.P. Rowe, C.J. Plant, and A. Bradfield. "Trials of the Anticoagulant Rodenticides Bromadiolone and Difenacoum against the House Mouse (Mus Musculus L.)" In: Journal of Hygiene 87.2 (1981), pages 171-177. DOI: 10.1017 / S0022172400069370 (cited on page 2).

[12] J. H. Greaves, D. S. Shepherd, and R. Quy. "Field Trials of Second-Generation Anticoagulants against Difenacoum-Resistant Norway Rat Populations". In: J Hyg (Lond) 89.2 (Oct. 1982), pages 295-301. ISSN: 0022-1724. pmid: 7130704 (cited on page 2).

[13] Ahmed Hodroge et al. "Biochemical Characterization of Spontaneous Mutants of Rat VKORC1 Involved in the Resistance to Antivitamin K Anticoagulants". In: Arch. Biochem. Biophys. 515.1-2 (Nov. 2011), pages 14-20. ISSN: 1096-0384. DOI: $10.1016 / \mathrm{j}$. abb. 2011.08 .010 . pmid: 21907178 (cited on page 2).

[14] J. Shang et al. "Zucker Diabetic Fatty Rats Exhibit Hypercoagulability and Accelerated Thrombus Formation in the Arterio-Venous Shunt Model of Thrombosis". In: Thrombosis Research 134.2 (2014), pages 433-439. DOI: $10.1016 / j$.thromres . 2014.04 .008 (cited on pages 2, 5).

[15] J.M. Siller-Matula et al. "Interspecies Differences in Coagulation Profile". In: Thrombosis and Haemostasis 100.3 (2008), pages 397-404. DOI: 10.1160 / TH0 8 02-0103 (cited on page 2).

[16] P.J.M. Salemink, J. Korsten, and P. de Leeuw. "Prothrombin Times and Activated Partial Thromboplastin Times in Toxicology: A Comparison of Different Blood Withdrawal Sites for Wistar Rats". In: Comparative Haematology International 4.3 (1994), pages 173-176. DOI: 10.1007 /BF 00798360 (cited on page 2).

[17] K.G. Mann, K. Brummel, and S. Butenas. "What Is All That Thrombin For?" In: Journal of Thrombosis and Haemostasis 1.7 (2003), pages 1504-1514. DOI: 10 . $1046 / j .1538-7836.2003 .00298 . x$ (cited on page 2).

[18] K.G. Mann. "Is There Value in Kinetic Modeling of Thrombin Generation? Yes". In: Journal of Thrombosis and Haemostasis 10.8 (2012), pages 1463-1469. DOI: $10.1111 / j .1538-7836.2012 .04799 . x$ (cited on page 2). 
[19] Maria del Pilar Huby et al. "Establishment of Methods for Performing Thrombelastography and Calibrated Automated Thrombography in Rats". In: Shock 42.1 (July 2014), pages 27-30. ISSN: 1073-2322. DOI: $10.1097 /$ SHK . 0000000000000163 (cited on pages 2, 5).

[20] Sébastien Lefebvre et al. "Monitoring of Antivitamin K-Dependent Anticoagulation in Rodents - Towards an Evolution of the Methodology to Detect Resistance in Rodents". In: Pesticide Biochemistry and Physiology (2017). ISSN: 0048-3575. DOI: 10 . $1016 / j$. pestbp. 2017.02 .003 (cited on pages 2,3).

[21] H.C. Hemker and R. Kremers. "Data Management in Thrombin Generation”. In: Thrombosis Research 131.1 (2013), pages 3-11. DOI: $10.1016 / j$.thromres . 2012.10 .011 (cited on pages 2,3).

[22] G. Jourdi et al. "Association Rate Constants Rationalise the Pharmacodynamics of Apixaban and Rivaroxaban". In: Thrombosis and Haemostasis 114.1 (2015), pages 78-86. DOI: 10 . 1160/ TH14-10-0877 (cited on page 2).

[23] Y. Dargaud et al. "Global Haemostasis and Point of Care Testing”. In: Haemophilia 18 (SUPPL.4 2012), pages 81-88. DOI: $10.1111 /$ j . 1365-2516. $2012.02855 . x$ (cited on page 2$)$.

[24] A.S. Lawrie et al. "The Clinical Significance of Differences between Point-of-Care and Laboratory INR Methods in over-Anticoagulated Patients". In: Thrombosis Research 130.1 (2012), pages 110-114. DOI: 10 . $1016 / j$.thromres . 2011.08 .027 (cited on page 2).

[25] H.C. Hemker et al. "Calibrated Automated Thrombin Generation Measurement in Clotting Plasma”. In: Pathophysiology of Haemostasis and Thrombosis 33.1 (2003), pages 4-15. DOI: 10 . 1159/000071636 (cited on page 2).

[26] S.N. Tchaikovski et al. "Development of a Calibrated Automated Thrombography Based Thrombin Generation Test in Mouse Plasma". In: Journal of Thrombosis and Haemostasis 5.10 (2007), pages 2079-2086. DOI: $10.1111 / \mathrm{j} .1538-7836.2007 .02719 . \mathrm{x}$ (cited on page 3 ).

[27] J.-P. Hérault et al. "Effect of Clopidogrel on Thrombin Generation in Platelet-Rich Plasma in the Rat". In: Thrombosis and Haemostasis 81.6 (1999), pages 957960 (cited on page 5).

[28] Y. Dargaud et al. "Monitoring Platelet Dependent Thrombin Generation in Mice". In: Thrombosis Research 126.5 (2010), pages 436-441. DOI: $10.1016 /$ j.thromres.2010.08.007 (cited on page 5).
[29] V. Prima et al. "Impact of Moderate Blast Exposures on Thrombin Biomarkers Assessed by Calibrated Automated Thrombography in Rats". In: Journal of Neurotrauma 30.22 (2013), pages 1881-1887. DOI: 10 . 1089 / neu. 2012.2758 (cited on page 5).

[30] T.-Q. Cai et al. "Factor XII Full and Partial Null in Rat Confers Robust Antithrombotic Efficacy with No Bleeding". In: Blood Coagulation and Fibrinolysis 26.8 (2015), pages 893-902. DOI: 10 . 1097 / MBC . 0000000000000337 (cited on page 5).

[31] M. Chitlur. "Challenges in the Laboratory Analyses of Bleeding Disorders". In: Thrombosis Research 130.1 (2012), pages 1-6. DOI: $10.1016 / j$.thromres . 2012.03 .011 (cited on page 5).

[32] H.C. Hemker et al. "Thrombin Generation, a Function Test of the Haemostatic-Thrombotic System". In: Thrombosis and Haemostasis 96.5 (2006), pages 553561. DOI: $10.1160 /$ TH06-07-0408 (cited on page 5).

[33] C. Petterino and B. Paolo. "Toxicology of Various Anticoagulant Rodenticides in Animals". In: Veterinary and Human Toxicology 43.6 (2001), pages 353-360 (cited on page 6).

[34] Toshio Harauchi et al. "Liver and Plasma Levels of Descarboxyprothrombin (PIVKA II) in Vitamin K Deficiency in Rats". In: The Japanese Journal of Pharmacology 40.4 (1986), pages 491-499. DOI: 10 . 1254 / jjp. 40.491 (cited on page 6). 